\title{
Le théâtre et l'ennui : Shakespeare serviteur de deux maîtres
}

\section{Dominique Goy-Blanquet}

\section{Q OpenEdition}

1 Journals

\section{Édition électronique}

URL : http://journals.openedition.org/shakespeare/1050

DOI : 10.4000/shakespeare.1050

ISSN : 2271-6424

Éditeur

Société Française Shakespeare

\section{Édition imprimée}

Date de publication : 1 novembre 2007

Pagination : 147-164

ISBN : 2-9521475-3-1

\section{Référence électronique}

Dominique Goy-Blanquet, "Le théâtre et l'ennui : Shakespeare serviteur de deux maîtres », Actes des congrès de la Société française Shakespeare [En ligne], 24 | 2007, mis en ligne le 30 mars 2010, consulté le 20 avril 2019. URL : http://journals.openedition.org/shakespeare/1050 ; DOI : 10.4000/

shakespeare.1050

Ce document a été généré automatiquement le 20 avril 2019.

(c) SFS 


\title{
Le théâtre et l'ennui : Shakespeare serviteur de deux maîtres
}

\author{
Dominique Goy-Blanquet
}

1 C'est officiel, le directeur du Printemps des Poètes vient de l'annoncer à la presse : « La poésie ne fait plus peur ${ }^{1}$ ». Est-ce une bonne ou une mauvaise nouvelle? Je ne sais pas. Faut-il le croire ? Je ne crois pas. Il parle en homme politique qui se veut rassurant - nous avons neutralisé une peste aussi barbare que la grippe aviaire ou le chikungunya. Une chose au moins est certaine, il confirme le caractère redoutable de la poésie.

\section{Les clés du royaume}

2 L'origine de cette peur est sans doute à chercher dans la nuit des temps, terreur sacrée de même nature que celle qui entourait les rois magiciens, les prêtres sorciers, les fous oraculaires. Nous avons surmonté nos craintes ancestrales en inventant l'asile, la guillotine et la laïcité, sans trouver le traitement ad hoc pour venir à bout des poètes. Les mettre en pièces comme jadis Orphée ou les exclure de la cité comme le conseillait Platon serait incorrect. Dans les années 80, on leur concéda un enclos en sous-sol au cœur de la ville : la Petite Salle de Beaubourg, où la Revue parlée de Blaise Gautier accueillit toute une génération de poètes français ou étrangers lors de messes basses dignes des catacombes.

Il se trouve que Blaise Gautier était le fils, mais le fils non reconnu, d'un homme de théâtre, Michel Saint-Denis. Invité un jour à Londres par son père, il avait rencontré Vivien Leigh et Laurence Olivier alors en pleine gloire, rencontre dont il gardait un souvenir ébloui, me confia-t-il un soir à l'issue d'une lecture. De là nous vint le projet d'éditer les trésors pieusement conservés de ce père lointain, si lointain que même un spécialiste comme Irving Wardle ignorait la filiation. Car Michel Saint-Denis était un peu oublié en France, alors qu'il a fondé un monument en Angleterre, avec Peter Brook et Peter Hall, la Royal Shakespeare Company. Mais Blaise quitta la scène avant de lui rendre son hommage. 
4 À l'époque des mélodies en sous-sol, le nom d'un poète s'affichait partout dans Paris : Shakespeare venait de dépasser Molière au hit parade des auteurs les plus joués en France. Ce poète que Victor Hugo brandissait jadis comme la foudre contre les philistins de l'ancienne critique, ce poète-là au moins ne faisait plus peur à personne. Toute une génération rééduquée par Jean Vilar lui amenait en troupe les enfants des écoles. Je l'ai fait aussi, et revois encore avec bonheur le visage outré d'une étudiante picarde à l'entracte du Richard II de Mnouchkine - «Mais c'est complètement faux, n'est-ce pas, Madame?»-, totalement conquise à l'issue du spectacle. Depuis Chéreau, tout jeune metteur en scène se devait d'affronter le continent Shakespeare pour conquérir ses palmes. À tel point que la revue esprit m'avait chargée d'organiser une rencontre avec Mesguich and Co. pour débattre de ce phénomène. J'ai envoyé mes invitations en leur proposant pour thème de discussion, "Shakespeare, poète de l'Angleterre ". Tous ont décliné, au motif résumé en ces termes par l'un d'entre eux : «La poésie c'est pas mon truc ». J'ai donc reformulé le projet en supprimant le mot poète, et ils sont venus. La rencontre fut brillante, portée par la créativité de leur rapport à Shakespeare, qu'ils élirent à l'unanimité forain en chef, premier saltimbanque de la profession : il suffisait d'opérer le poète de la poésie pour lui offrir les clés de la ville, ou du moins de la forteresse théâtrale².

5 Un abîme s'était ouvert devant moi, dont j'étais loin encore de mesurer l'ampleur. Car les expériences du type Esprit ne se sont pas limitées à la scène française, ni à la scène tout court. L'exclusion fonctionnait dans les deux sens. Nombre de poètes se refusaient à parler aux foules, la poésie devait rester pour chacun une relation privée, secrète, avec son lecteur. Les litanies chuchotées de Beaubourg étaient déjà pour certains une grande concession aux modes orales importées d'Amérique. Aujourd'hui encore, nombre de poètes acceptent de lire tout haut mais marquent leur différence en refusant toute théâtralité, tout comme ils refusent de confier leurs œuvres à des acteurs. Lesquels acteurs, s'ils mettent la main ou la voix sur un poème, tendent à lui impulser une dramaturgie et un style déclamatoire pas toujours bien venus. Ainsi lorsque ce siècle eut deux ans, anniversaire oblige, j'ai entendu coup sur coup un Hugo vieilli par l'emphase d'un Comédien-français - « Ôôô, soldats de l'An II ! ôôôôô guerres ! éééépopées ! - puis un Hugo bien vif, retrouvant dans la voix d'un poète le battement du vers et la fraîcheur de ses jeunes «Soldats»: «Sans repos, sans sommeil, coudes percés, sans vivres, / Ils allaient, fiers, joyeux..."

Quelque temps après la réunion d'Esprit, lors d'une interview avec Deborah Warner à propos de son superbe Richard $I^{3}$, j'ai abordé un passage qui m'avait paru rétréci par la posture adoptée, «Let us sit upon the ground». Au beau milieu de la tourmente, le roi fige l'action sur l'image de la couronne creuse où sombre la monarchie. Fiona Shaw suçant son pouce enfermait cette scène cruciale dans la logique du rôle qu'elle interprétait, un enfant qui refuse de grandir. Justement, tentai-je d'argumenter, c'est là que le discours dépasse le personnage, que les mythes du pouvoir se dessinent vers à vers, et j'ai lâché le mot fatal. Il me valut à peu près la même réponse que celle de ses collègues français, «Poetry is not my thing ». Je suis passée à la double performance, magnifique, de Paola Dionisotti, parcours rectiligne de la rigide Duchesse de Gloucester, volutes de la Duchesse d'York encerclant les hommes de pouvoir de sa féminité. «Oh, so you liked Paola!» Apparemment cette manière de tracer dans l'espace les cadences de leur rhétorique propre étaient l'initiative de l'actrice, une "ancienne» que les autres trouvaient un peu « décalée ». J’ai senti le différend se creuser. Il me rappelait une plainte 
fréquemment entendue l'été dans les jardins de Stratford: les acteurs de la RSC ne savent plus dire les vers. Ubi sunt Jacobi, Ashcroft, Gielgud, Scofield? Question de génération? Pas seulement. Moi aussi, malgré mes impatiences de Beaubourg, je commençais à m'ennuyer ferme au théâtre. Et après quatre heures de Hamlet écartelé par Lavaudant, à me convertir aux vertus de l'opéra, où le tempo tient en respect le narcissisme du chef d'orchestre.

\section{Joutes académiques}

7 Est-ce une coïncidence ? Oxford University Press venait de remettre à sa place le poète puissant et solitaire en donnant priorité à la création sur scène de son œuvre. Coup d'envoi d'une révolution des pratiques éditoriales, que le TLS résuma en un titre, « Hamlet by Dogberry ${ }^{4}$ ». S'agissant de Shakespeare, il y a comme toujours derrière les positions de principe littéraires des enjeux idéologiques. Gary Taylor rappelle en ouverture d'édition que le théatre fut de tout temps en butte à l'hostilité des autorités. Ce qu'il ne dit pas, c'est que l'ennemi n'a plus rien à voir avec ces pères puritains qui voulaient le chasser hors de la cité, bien au contraire, il a pris la direction de l'entreprise, et les conflits sont des conflits de pouvoir. Entre maisons d'édition. Entre écoles rivales à Cambridge, véritable pépinière d'acteurs et de metteurs en scène. Entre ces acteurs-là et les autres. "To hell with Oxford and Cambridge » déclare Peter Brook, lui-même diplômé d'Oxford, qui soutient avoir lu au maximum trois ouvrages sur Shakespeare, et se moque gentiment de Peter Stein, qu'un excès de lecture paralyse ${ }^{5}$. Si Peter Hall a passé sa jeunesse dans les bibliothèques ${ }^{6}$, Deborah Warner, disciple de Brook, dit n'avoir pas besoin de livres mais d'acteurs : "plays called political by academics need reassessment ». Et Fiona Shaw de rappeler comment « For a long time, universities owned the plays », laissant entendre que désormais Shakespeare est revenu parmi les siens ${ }^{7}$.

Peter Hall fit ses premières classes théâtrales avec l'anti-Leavisite George Rylands, même s'il allait en catimini écouter les conférences du «new critic » Frank R. Leavis, qui n'avait pas une grande passion pour le théatre. Leavis «thought of the theatre as a relatively frivolous place » confie Trevor Nunn, un autre de ses étudiants. Nunn prendra bientôt avec Hall les commandes du théâtre subventionné ${ }^{8}$, mais pour l'instant c'est Rylands qui joue un rôle pivot: survivant du Bloomsbury Group, il est tout à la fois professeur de littérature à King's College, administrateur d'une des plus grosses entreprises théâtrales $\mathrm{du}$ West End, H. M. Tennent, et metteur en scène de la Marlowe Society à Cambridge où il maintient «a strong tradition of verse-speaking ». Hall raconte dans son autobiographie comment Rylands « directed with his nose firmly in the text. He was more concerned with our line-endings and our iambics than with whether we were bumping into each other. John Barton compensated with being in charge of the battles ${ }^{9}$ ».

Rien de comparable à ces érudits praticiens en France, où l'université a longtemps traité les textes dramatiques comme n'importe quel texte littéraire, et se souciait peu de les faire jouer. Il fallut les travaux pionniers d'un Jean Jacquot au CNRS à partir des années 50 pour qu'elle prenne en compte les arts du spectacle. Aujourd'hui l'université et le théâtre français se disputent Shakespeare. S'ils acceptent de dialoguer, c'est souvent à fleurets mouchetés. Certains membres de la Société Shakespeare se rappelleront Antoine Vitez rue d'Ulm ripostant aux questions professorales et aux critiques adressées à son traducteur en évoquant un Hamlet de jadis interprété par un troupeau d'oies savantes. Ou Peter Brook faisant marcher son auditoire à la baguette et lui interdisant toute question 
lors d'un colloque au Sénat. Je me suis demandé à ce moment-là qui d'autre en France exerçait un pouvoir aussi absolu.

Shakespeare, conquis sur les conservateurs du poème par les artisans du drame, était repassé du texte à la scène. Passeur résolu du théâtre, Gary Taylor va droit au point sensible en comparant les deux versions de Richard III, Q et F : «most of the material present only in $\mathrm{F}$ consists of static poetic elaboration which slows up the dramatic pace. » Or, précise-t-il, «F seems a text closer to the author's original composition than $\mathrm{Q}$, which by contrast shows clear signs of theatrical provenance ». La plus brève, $Q$, choisie pour base de l'édition Oxford, « has both suffered and benefited from direct contact with the theatre ${ }^{10}$ ", c'est-à-dire l'intervention des comédiens, que Shakespeare a eu la sagesse d'accepter: «We find it easier to believe that Shakespeare on reflection intelligently cut such elaborations than that he so unintelligently padded out a play already taxingly long. » À Cambridge, l'éditeur du First Quarto les inscrit au chapitre intitulé «Actors' 'Improvements' «, améliorations cernées de guillemets et qualifiées par ce commentaire: "There can be little doubt that what Shakespeare drafted appears in $F$ and that $Q$ prints what was performed by the two actors - the two clowns, Kemp and Cowley ${ }^{11}$.

11 À l'examen, certains des passages incriminés n'ont rien de poétique, ils sont simplement verbeux. Mais parmi les rembourrages figurent aussi le plaidoyer incestueux de Richard à Elizabeth, ou l'apostrophe à la Tour, «Pity, you ancient stones... » auxquels on renonce moins aisément ${ }^{12}$. John Jowett dans son édition de Richard III pour Oxford leur trouve un thème commun, "the begetting, presence, or killing of children. These passages are significant to a reading of the play that stresses its moments of pathos ", mais poursuit-il, «From another point of view these passages clog the play with sentiment and slow its action $^{13}$. »Clog, comme le cholestérol dans les artères.

\section{Haut les chœurs !}

Il faut dégraisser le mammouth. Dans l'offensive de dégraissage, c'est souvent le rôle autoritaire des « profs » gardiens du texte qui est visé. Les éditeurs d'Oxford s'attaquent d'abord au conservatisme de collègues rivaux en donnant leur préférence au texte allégé par le travail à la scène. Est-ce à dire pour autant que des comédiens en groupe sont forcément plus créatifs, plus talentueux qu'un auteur à lui seul ? Sans vouloir minimiser leur apport, ça se discute. Leur jeu peut produire des éclairs d'illumination sans égal, mais depuis que j'ai entendu l'un d'eux aux Bouffes jurer par Cérébos, j'avoue mieux comprendre l'irritation de Hamlet contre les clowns. Est-ce bien le rôle des éditeurs de donner priorité à leur version? Or nombre de «Complete Shakespeare » reproduisent le texte d'Oxford sans avertir les lecteurs de leurs choix éditoriaux, à commencer par une édition grand public comme Laffont Bouquins, ou Norton qui vise le marché étudiant ${ }^{14}$.

13 Après avoir réagi par un chapitre intitulé «Down with poets!», que la maison Oxford, très sport, a publié sans retouche ${ }^{15}$, j'ai mis la question des lenteurs poétiques procrastination? - au programme de l'atelier «Le Poète dans la Cité », en ajoutant aux propos cités plus haut celui d'Antony Hammond, qui traduit bien le dilemme :

to distinguish between "Shakespeare » the solitary artist, scribbling in his garret (as it were), and "Shakespeare" as the complex of author/book-keeper/actor/ prompter and others who actually created the play in the sense of a living performance on the stage is to find oneself on one side or other of a philosophical 
issue concerning the nature of artistic creativity. Each position has its justification ${ }^{16}$

$\ldots$

14 Curieusement, tous les spécialistes de Performance Studies invités à l'atelier se sont récusés, y compris ceux qui avaient accepté l'invitation au départ. J'ai insisté, craignant de voir l'ouvrage dériver vers une attaque en règle des comédiens qui n'était nullement mon propos, plaidé pour l'amour du théâtre, sans succès : ils avaient tous un sanglier sur le feu, trois livres en chantier, des cours, des colloques. La présence du théâtre dans l'ouvrage est heureusement assurée par le critique Michael Coveney, un passionné qui a suivi depuis ses débuts une entreprise citoyenne, les Glasgow Citizens, et par un passeur de frontières à qui j'avais demandé de tenir son carnet de route: Jacques Bonnaffé l'acteur des poètes, interprétant les deux Antipholus face à l'acteur comique Bernard Menez, atteste de la division des champs. Cette Reprise des Erreurs discutée au colloque 2005 sur « Shakespeare et le jeu » était une somme de contradictions échevelée, une pâte textuelle dopée par des injections de Quignard et autres gags scéniques.

Le poète Dominique Buisset, helléniste et traducteur, ouvre le chantier avec un OMNI (Objet Mimétique Non Implantable), le "stasimon", danse sur place qui impose un suspens à l'action héroïque : « au moment où la cité athénienne "invente la démocratie", l'entrée du chœur lyrique dans le théâtre, c'est l'entrée du collectif dans la mimésis : les héros ne sont plus seuls. » Ainsi, «de l'épopée à la tragédie, il y a comme une mise en ellipse du héros, un déclin de l'individu, une perte de souveraineté17. ${ }^{17}$ Dans L'Tliade, qui s'appuie sur des valeurs aristocratiques, les reproches d'un Thersite à Agamemnon ne provoquent que mépris et hilarité chez ses auditeurs. La tragédie met en cause ces valeurs et la valeur de l'action héroïque, oppose le chœur passif mais bavard au héros fragilisé, inscrit pour la première fois le mot et le fait de la démocratie dans la littérature : dèmou kratoussa keir, "Dis-nous [...] de quel côté la main souveraine du peuple se lève en majorité18. " Assemblé sur les gradins du théâtre, le peuple assiste à sa propre mimésis. Cette tension fondatrice, dialectique, entre mouvement et immobilité, il serait vain de chercher à la résoudre par une victoire de l'action sur les "assaisonnements » de la poésie. D'autant que la riche variété des mètres lyriques est réservée aux chants du chœur, le héros doit se contenter de la simplicité iambique.

Quand et pourquoi Shakespeare choisit-il de faire long au lieu d'aller droit au but ? Brian Gibbons plante vaillamment ses crampons dans la paroi rhétorique pour sonder les aspérités qui diffèrent le dénouement, réserve de richesses où les adaptateurs successifs ont pu tailler à volonté ${ }^{19}$. Le chœur n'a pas vraiment disparu du drame shakespearien, il investit les personnages et s'insinue partout, en lisière des consciences. Au fond, priver l'auditoire de "static poetic elaboration" constitue un véritable contresens politique, puisque le chant sur place amorçait un transfert de souveraineté vers le peuple. Ce transfert au sein de la cité, et ce qu'il en coûte aux valeurs héroïques, Coriolan l'incarne sous nos yeux. Quant à Thersite, il ne fait plus vraiment rire dans Troilus et Cressida, les héros ressemblent trop à leur caricature. Shakespeare ne ménage pas Homère quand il le convoque au tribunal du théâtre. Hécube et sa douleur inconsolée hantent la scène, de Lucrèce à Hamlet, comme le rappelle Richard Marienstras ${ }^{20}$.

\section{Le veilleur de nuit}

17 Sur les remparts d'Elseneur, le suspens prend une dimension métaphysique, l'hésitation se tient « au seuil de la seule chose qui nous préoccupe, l'après » : ce soi-disant retard à 
l'action, écrit Jacques Darras, "qui ne sait tout au fond de lui qu'il s'agit du seul ralentissement propice à poser la seule question qui vaille: la question du seuil mortuaire. Un rêve? Un sommeil ? Rien ${ }^{21}$ ?» En cours de dialogue ou d'intrigue, Shakespeare laisse vibrer les images du monde, dans une forme d'engourdissement, sur le revers du tissu: le monde comme activité rêvée, «fabric » où nous sommes rêvés par quelqu'un d'autre, "stuff as dreams are made on». Il dit que la passivité a valeur d'initiation - nous ne serons acteurs que si nous savons être spectateurs. Prospero le magicien donne congé à Ariel et aux elfes des collines avant de regagner son duché. La poésie introduit du retard dans le mouvement du monde, elle ne peut être montrée que quand l'action se brise, dans le dessaisissement. Y ont accès ceux qui sont exclus de l'activité sociale. Les rois la mettent au service de l'action; les plus fragiles, ceux qui abdiquent, sont les plus poètes. Pour Yves Bonnefoy, la poésie est reprise, ressaisissement par la mémoire, réflexion critique sur notre activité. Dans le discours ordinaire, elle est en exil par rapport à la réalité, raison pourquoi selon lui le théâtre de Shakespeare devient grand à partir de Hamlet, lorsque le poète pense activement ce que peut être la poésie dans son essence ${ }^{22}$.

Si Hamlet fascine les écrivains, c'est que la pièce est un poème de bout en bout, de la première question inquiète, "Who's there ? ", à la fin de l'interrègne, « Good night, sweet prince ». Horatio le sceptique ébranlé conjure l'esprit de la lumière, « the god of day », le gardien Marcellus invoque la sainte nuit de Noël, lorsque la relève du matin vient soulager leur veille oppressante : «But look, the morn in russet mantle clad / Walks o'er the dew of yon high eastward hill. » Une chaîne d'images dramatiques s'ouvre et file dans le sillon de leur incertitude jusqu'à la victoire de "proud Death" conviant tous les acteurs du conflit à son festin cannibale. Cette fois c'est le héros en personne qui suspend et s'interroge sur le bien-fondé de l'action, qui s'aventure aux confins du sommeil éternel. Hamlet convoqué pour affronter le fantôme saisit le monde dans un regard d'une acuité sans faiblesse, le rêve poétiquement et le renvoie réfléchi en profondeur à la cour grossière, primaire, de Claudius. C'est Polonius, rappelons-le, qui décrète que « Brevity is the soul of wit ", et demande aux comédiens d'abréger, simplifier, schématiser la matière rêveuse du poème. Ordre que maints batteurs d'estrade exécutent aujourd'hui sans réflexion, prévenant nos désirs et nos appétits. "Trust the text », conseille pourtant Peter Hall. « If it is written by a master, it works like yeast on the audience's imagination ${ }^{23}$. »

19 La crainte d'ennuyer trahit un pathétique et souvent désastreux manque de confiance dans le poème: au lieu d'écouter ses images, nos diététiciens aux abois abrègent, psychanalysent le surnaturel, parquent l'imaginaire dans une logique prosaïque. Les plus inquiets font donner les clowns pendant que le texte défile, ou emballent la précision des mots dans une dynamique ronflante, rabattent les héros sur le monde sans foi ni loi du business. Lear revu par Engels est mis en minorité dans son entreprise à peine publique. Le dernier Richard III en date mouillait sa chemise, écrasant les feintes des joutes verbales par une véhémence uniforme : le public bon prince a applaudi l'athlète ${ }^{24}$.

Allez voir Hamlet, recommande de son côté Darras, « et sortez immédiatement si, dès le premier acte, le fantôme ne vous fait pas peur ». Mais qui ose encore croire et faire croire aux fantômes? Le cavalier de Chéreau, surgi des ténèbres sur son dangereux pur-sang, réussissait le pari impossible de nous projeter dans le monde des esprits. Lavaudant a préféré couper la progression du spectre en sous-sol. Marthouret en réduisant son Hamlet aux scènes d'action en a fait paradoxalement le plus lent du répertoire. Brook aux Bouffes du Nord l'a donné en version originale parce que « la vie de la pièce est contenue dans les 
mots de Shakespeare. Leur sonorité est essentielle à la structure de la pièce ${ }^{25}$. » À noter qu'il ne s'est guère soucié auparavant des sonorités du français : sa Tempête était un pur chef d'œuvre, si on se résignait à perdre la moitié d'un texte déformé par les dictions étrangères, son ami Peter Stein s'est empressé de le souligner. Quant au Hamlet du nouveau millénaire, inutile même de chercher à comprendre la tirade d'Hécube, elle était traduite en orghast. Le reste de la pièce, ce qu'il en restait, se voulait une épure : «Le découpage est inévitablement le reflet d'un goût des spectateurs de son temps. Ce que l'on a enlevé, c'est ce qui n'est plus nécessaire ${ }^{26}$. » Jugement terrible, derrière la douceur de l'expression, sur les spectateurs que nous sommes, aussi primaires que l'entourage du prince, un svelte gymnaste, était devenu transparent. L'épure, la vraie, inspirée à tous les sens du terme par les fantômes de Hamlet c'était le spectacle précédent, Qui est Là?

\section{Hugo ou Artaud?}

21 Qui nous rêve aujourd'hui ? C'est la question. En moins d'un siècle on a vu s'opérer un renversement complet de situation. Les poètes anglais qui redécouvraient Shakespeare grâce aux leçons de Schlegel se méfiaient du théâtre autant que les poètes français d'aujourd'hui. Coleridge en tête tenait Richard II pour un poème bien trop subtil pour les grossièretés de la scène et les capacités d'un acteur. Il fallut la performance d'un Charles Kean et les éloges de l'écrivain Walter Pater pour inverser la tendance : «In the hands of Kean the play became like an exquisite performance on the violin ${ }^{27}$ ». Le Richard élégiaque l'emporta alors sur le Richard politique plus combattif des générations antérieures. C'est le même Pater qui le couronne "exquisite poet ", noyant les tensions entre drame et poésie dans la métaphore musicale. Les poètes romantiques ne l'avaient pas attendu pour choisir Richard comme roi d'élection, et nimber Shakespeare d'une autorité quasi divine.

En France, la bardolâtrie a cheminé par les lettres avant de gagner l'ensemble des arts, et provoqué d'abord des querelles entre écrivains. Copeau avait quelques bonnes raisons de vouloir arracher Hamlet à l'hamlétisme. À la décharge des acteurs, il faut dire que les poètes ont eux-mêmes créé le malentendu sur Shakespeare en communiant dans le culte de l'homme-océan, l'ivresse du sauvage. Hugo veut reprendre Shakespeare au trop classique Voltaire, et à François Guizot le monarchiste. Quand paraît la traduction de François-Victor, il lui envoie un petit mot affectueux :

Je me suis occupé de toi. J'ai vu Joly qui va enfin te payer sa dette, et a esquissé devant Charles et moi le plan de son article sur ta traduction qu'il déclare admirable. Il compte te comparer à Guizot et le massacrer, pour le punir d'avoir, lui doctrinaire et classique, touché à Shakespeare ${ }^{28}$.

Les deux traducteurs proposent deux lectures radicalement opposées de Richard II. Pour Guizot, la pièce est royale, «la doctrine du droit divin y est sans cesse présentée accompagnée de cet intérêt que font naître le malheur et le spectacle de la grandeur déchue ${ }^{29}$. " Pour Hugo fils, c'est un hymne au Parlement, où Shakespeare célèbre l'« insurrection heureuse de l'opprimé contre l'oppresseur »: "Devant ce misérable Westminster où l'on divinise la tyrannie, il élève subitement le formidable Westminster où on la détrône ${ }^{30}$.» Entre temps, Baudelaire est monté au créneau, avec non moins de vigueur, lors du tricentenaire de Shakespeare que le clan Hugo s'approprie abusivement, hurle-t-il dans les colonnes du Figaro: 
Parlons un peu du vrai but de ce grand jubilé. Vous savez Monsieur qu'en 1848 il se fit une alliance adultère entre l'école littéraire de 1830 et la démocratie, une alliance monstrueuse et bizarre. Olympio renie la fameuse doctrine de l'art pour l'art, et depuis lors, lui, sa famille et ses disciples n'ont cessé de prêcher le peuple, de parler pour le peuple, et de se montrer en toutes occasions les amis et les patrons du peuple. «Tendre et profond amour du peuple! » Dès lors, tout ce qu'ils peuvent aimer en littérature a pris la couleur révolutionnaire et philanthropique. Shakspeare est socialiste. Il ne s'en est jamais douté mais il n'importe... Nous sommes familiarisés avec ce genre de supercherie ${ }^{31}$.

En revanche pas de conflit armé à l'époque entre hommes de lettres et forains. Hugo père a lui-même, comme dirait Gary Taylor, «both suffered and benefited from direct contact with the theatre ", en exposant Hernani aux règles du spectacle et à la censure de Mademoiselle Mars $^{32}$. Jacques Copeau (l'oncle de Michel Saint-Denis) collabore avec Gide à la création de la Nouvelle Revue Française. Tous les poètes de leur génération traduisent ou relisent Shakespeare, qui simultanément prend une place croissante sur la scène. Gallimard rue Sébastien-Bottin est proche du Vieux-Colombier, surnommé l'«annexe théâtrale » de la NRF, où Antonin Artaud émettra ses derniers balbutiements. Lequel Artaud fournira les arguments de la rupture qui va suivre, en légitimant toutes les formes d'intervention sur la chair des textes.

L'art de la coupe est une vieille obsession chez les interprètes de Shakespeare : «Coupez, taillez, rognez, surtout effacez», conseillait Voltaire, à l'instar de Dryden et autres tailleurs anglais $\mathrm{s}^{33}$. Au $\mathrm{xx}^{\mathrm{e}}$ siècle, dans les pas d'Artaud, la mise en pièces de l'auteur devient partie intégrante du rituel théâtral, ce que Gary Taylor décore d'un bel euphémisme, la socialisation du texte, « its progress from a private to a socialized mode ${ }^{34}$ ». "Briser le langage pour toucher la vie, c'est faire ou refaire le théâtre ${ }^{35}$, " clame Artaud: "On doit en finir avec cette idée des chefs d'œuvre réservés à une soi-disant élite, et que la foule ne comprend pas ». Premier accusé ? "Shakespeare lui-même est responsable de cette aberration et de cette déchéance ». Le verdict est sans appel : «La poésie écrite vaut une fois et ensuite qu'on la détruise. Que les poètes morts laissent la place aux autres ", car "c'est notre vénération devant ce qui a été déjà fait, si beau et si valable que ce soit, qui nous pétrifie, qui nous stabilise ». Décidément la stabilité inquiète! Artaud conclut qu'il y a un risque dans son programme de mise en transe, « mais je propose quelque chose pour sortir du marasme, au lieu de continuer à gémir sur ce marasme et sur l'ennui, l'inertie et la sottise de tout ${ }^{36}$.» Puis il enchaîne sur «Le théâtre et la cruauté ».

Quand Peter Brook tourne la déclaration d'indépendance d'Artaud en machine de guerre contre l'establishment théâtral, il rompt avec la grande tradition académique. Sous son égide, la Royal Shakespeare Company entame une «Theatre of Cruelty season » et une longue ère de mutilations expérimentales ${ }^{37}$. Hall se souviendra plus tard non sans gêne «I blush at our frenzy of adaptation » - des coupes sauvages infligées à Henry vi par lui et John Barton pour The Wars of the Roses:

Once you start monkeying with original texts, either as a translator or an adaptor, you start to put on the creative cloak yourself. And it soon becomes easy to believe that you have a right to «improve » Shakespeare... Most of the cutting and editing and rewriting of the classics occurs because we don't understand what the original is doing or - even more likely - are incapable of realising it ${ }^{38}$.

Barton son complice de l'époque s'est fait depuis taxer d'« iambic fundamentalism ${ }^{39}$ ». On est loin de l'assurance qu'ils exprimaient du temps des Roses en compensant les coupes par 1400 vers de leur cru, "hardly distinguishable from Shakespeare's ", selon eux ${ }^{40}$. 
Brook, on l'a vu, ne partage pas ces remords. Il n'a pas à se justifier, ses spectacles sont parfaits dans leur genre, ils parlent ici maintenant. Mais imaginez un instant que Hamlet passe à la postérité en version orghast ?

\section{L'amour poète}

Intelligent ou pas, soumis ou non devant les Bottom et Dogberry de sa troupe, Shakespeare fait preuve d'une indéniable humilité en invitant à se défier des artistes en général, ces singes de la nature, et plus particulièrement des gens de son espèce. Peu de références à la poésie dans son œuvre, la plupart négatives. Le sycophante anonyme de Timon: « Art not a poet? - Yes. - Then thou liest. » Cinna le poète mis en pièces par les Romains, tel Orphée déchiré par les Bacchantes. Dans le Songe, Thésée range le poète avec dédain parmi les fous et les amants lunatiques, mais pour une fois c'est lui l'idiot qui n'a rien vu rien compris. La question de fond est confiée, comme souvent, à une voix des plus naïves, la jeune paysanne Audrey : «I do not know what 'poetical' is. Is it honest in deed and word? Is it a true thing? » La réponse va au cœur du paradoxe : « the truest poetry is the most feigning ${ }^{41} »$. Hugo fils résume admirablement le dilemme dans son Introduction aux Sonnets :

Le faiseur de sonnets devait s'imposer, comme amant, des règles plus rigoureuses que comme poëte. Après avoir subi les exigences de la rime, il fallait qu'il subît patiemment les cruautés de la belle ; il fallait qu'il continuât sans relâche de courir après l'une comme après l'autre, avec cette condition de toujours manquer la belle et de ne jamais manquer la rime ${ }^{42}$.

Malgré cet éclair lucide, il ne peut s'empêcher d'y chercher matière dramatique. Au lieu des antithèses, concetti, images, sons, rimes et rythme admirés par son formidable technicien de père, il se concentre sur le mystère des Sonnets dont il bouleverse l'ordre pour les constituer en séquence narrative. Le sonnet 71 « No longer mourn» devient le 145, au terme du parcours :

Les sonnets de Shakespeare contiennent en effet tout un drame. Exposition, complications, péripéties, dénoûment, rien ne manque à ce drame intime où figurent trois personnages : le poëte, sa maîtresse et son ami [...] : ce n'est plus William Shakespeare, c'est Will que nous voyons [...] Ce n'est plus l'homme public, c'est l'homme ${ }^{43}$.

Mais François-Victor a dû sentir la discordance entre ces deux commentaires car le premier disparaît de la réédition de 1880. Dommage : il posait très simplement, très clairement, la question de la posture en poésie. Depuis Wordsworth, on a usé en vain tout un trousseau de clés sur le cœur de Shakespeare, en oubliant que le cœur du sonnettiste fait partie de son équipement de base : « Fool, said my Muse to me, look in thy heart and write $^{44} »$. Wyatt dans la pose de l'amoureux éconduit exprime la souffrance infligée par la froideur de sa dame et les conventions du genre, aux antipodes de sa nature vigoureuse. Sidney dédie Astrophel non à sa muse mais à son épouse. Spenser bouscule les codes courtois en célébrant le mariage. Dernier sur les rangs, Shakespeare invente une variation nouvelle en faisant de la belle lointaine un beau. Qu'il ait fourni ou non la clé d'un drame personnel, peu importe, c'est à un drame plus vaste qu'il est confronté, non une déclinaison de toutes les orthographes possibles de mètre ou master mistress, mais la division centrale de son œuvre, théâtre et poésie, l'action ou ses assaisonnements, sa mise en vacance. La Pléiade vient de consacrer la rupture en excluant Shakespeare de l'anthologie de poésie ${ }^{45}$. Pourquoi ? Parce qu'il appartient à la section « Théâtre ». Exit le 
poète dramatique qui tenait bon depuis Aristote. Exit la principale source de la langue poétique anglaise.

Coupure que Shakespeare lui-même s'évertue à ignorer, en glissant des sonnets entiers et toute sorte de chants dans ses tirades, des dialogues dans ses sonnets. Le point le plus mystérieux de sa vie reste sans doute son rapport à la poésie, la liberté solitaire, royale, de la poésie, qu'il soumet aux contraintes du théâtre. Pourquoi ? Pas seulement pour l'argent. Il n'écrira pas de poésie dans sa retraite confortable. "The play's the thing... ». S'il revient au drame historique en fin de carrière, c'est pour poser une fois de plus, autrement, la question de la vérité. Henry $v$ convoquait « a muse of fire » dont les chants épiques étaient contredits ou au moins ébréchés par l'action guerrière. All is True met en miroir un jeu de masques vertigineux: « Now this masque / Was cried incomparable; and th'ensuing night / Made it a fool and beggar ${ }^{46}$. » L'art du spectacle s'autodénonce, torpille sa propre entreprise de séduction, comme la statue d'Hermione en s'animant réduit l'œuvre d'art du plus grand sculpteur à l'insignifiance. L'artiste Shakespeare qui cherche un accord idéal entre le beau et le vrai ne peut dire mieux. Le théâtre, cette mauvaise copie de la création divine dans sa complexité, la métaphore du théâtre, où la poésie fait irruption, cherchent la forme visible de cette concordance. "Le beau serviteur du vrai", selon l'expression de Victor Hugo ${ }^{47}$.

\section{Contre la division}

Acteur républicain? poète royal ? Le texte, le poème dramatique résiste à toutes les divisions. Hugo s'invente un Shakespeare révolutionnaire, cependant il note que chez lui la figure reine, c'est l'antithèse, cette "faculté souveraine de voir les deux côtés des choses $^{48}$ » - la figure que saint Augustin dans sa lecture dramatique de l'histoire chrétienne place au cœur de la création ${ }^{49}$. Rylands, après avoir rappelé que la prose dans le théâtre élisabéthain servait surtout aux personnages populaires et comiques, montre que Shakespeare brouille tous les usages, s'affranchissant du vers de Marlowe, le pliant au réalisme, et ennoblissant la prose ${ }^{50}$. Le vers blanc de Paradise Lost, où Chateaubriand reconnait la facture d'un républicain romain, c'est le pentamètre élisabéthain qui a « both suffered and benefited from direct contact with the theatre ». Son meilleur équivalent, estime Bonnefoy - même s'il ne l'utilise pas - c'est le vers de onze pieds : "Quand on le coupe après le sixième, il commence comme une indication de l'idéal, mais c'est pour s'achever, avec ces cinq syllabes qui ramassent et laïcisent, comme un fait ouvert à l'avenir d'autres faits ${ }^{51} »$.

Aristote n'a pas dit s'il préférait Homère ou Eschyle. Pour ma part, je ne connais pas de plus beau poème que l'ouverture d'Agamemnon, le veilleur sur le toit qui guette l'apparition de la lumière, je n'ai pas vécu d'expérience théâtrale, d'anagnorisis plus fortes qu'en suivant les Atrides de Mnouchkine quinze heures de rang. Mon irritation relève du dépit amoureux, j'ai adoré le théâtre, je souffre et je lui en veux de m'ennuyer en cherchant à me distraire de textes qui lui pèsent. Je crois profondément qu'il doit défendre sa place dans la cité. Et qu'on tue le malade en l'opérant de la poésie. Si Shakespeare reste unique, indispensable, c'est qu'il se tient aux antipodes du messianisme hugolien, qu'il offre la voix souveraine du poète à la multiplicité, à la vulgarité des voix, qu'il accepte la dissonance là où le désir d'harmonie conduirait à faire taire toute voix rivale. 
34 l'action et sa remise en cause, le héros et le chœur populaire, la cacophonie et son nécessaire protocole rhétorique, la confusion de «fair is foul » et les repères normatifs, c'est toute la question. "'Tis too long for a play » reste un argument imparable. Notre capacité d'écoute a changé, elle décline au rythme du découpage télévisuel. Notre éclatement en tribus, clans, sectes et communautés urbaines réduit à peu de choses les codes partagés auxquels peut encore se référer l'interprète. La vacuité actuelle du discours politique conforte notre résistance à la rhétorique, et voilà qu'elle revient masquée, dans la bouche de tribuns populistes démagogues... ou messianiques. La mission du théâtre a rarement été plus difficile, et plus vitale. Chers poètes, chers acteurs, écoutez-vous. Nous vous écouterons.

\section{NOTES}

1. Propos de Jean-Pierre Siméon cités par Cécile de Corbière, «Chanter dans les villes », Le Monde , 10 mars 2006.

2. "Meurtres dans un jardin français », entretien avec Daniel Mesguich, Stuart Seide, Georges Lavaudant, François Marthouret, Christian Colin, Bernard Sobel, L'Angleterre à contre-courant, ESPRIT, juillet 1985.

3. Janvier 1996, Maison de la Culture, Bobigny. Production du Royal National Theatre.

4. Brian Vickers, «Hamlet by Dogberry : A Perverse Reading of the Bad Quarto », Times Literary Supplement, 24 Dec. 1993, p. 5.

5. Notiert von Der Gliewe, «Duett der Titanen », Theater Heute, jan. 1991. Gerhard Stadelmaier, «Das Ballett der Königsdiener - Peter Brook und Peter Stein », Frankfurter Allgemeine, 13 nov. 1990.

6. Hall, op. cit., p. 50.

7. «Shakespeare : Les Rois dans la tempête », difusé par ARTE le 22 avril 1997, archives de l'I.N.A.

8. Lors d'une interview avec Jack Bradley sur sa mise en scène de The Cherry Orchard, National Theatre, 31 oct. 2000. Peter Hall fut directeur du National Theatre de 1973 à 1988, Trevor Nunn celui de la RSC de 1968 à 1986.

9. Hall, op. cit., p. 72-73.

10. Gary Taylor, «Richard III ", William Shakespeare: A Textual Companion, ed. Stanley Wells and Gary Taylor with John Jowett and William Montgomery, Oxford, 1987, p. 228, 230.

11. The First Quarto of King Richard III, ed. Peter Davison, The Early Quartos, Cambridge, C.U.P., 1996, Intro., p. 22. " The introduction shows how the actors' involvement helped to produce the text we have ", annonce sans s'engager la couverture, le but de cette série, souligné par son General Editor Brian Gibbons, étant de fournir des outils de travail aux chercheurs.

12. Pour une liste complète des variantes, voir John Jowett, éd. Richard III, Oxford, O.U.P., 2000, Appendices A-D, 359-85.

13. Jowett, op. cit., Intro., p. 131. Le texte retenu est celui $Q$.

14. On peut saluer l'initiative de la collection Pléiade en 2002, Shakespeare, Tragédies, qui présentait pour la première fois un auteur étranger avec le texte original en vis-à-vis de la traduction, texte établi par Gisèle Venet, alors que toutes les éditions bilingues auparavant se contentaient de reproduire une version britannique, Cambridge, Oxford ou Arden.

Actes des congrès de la Société française Shakespeare, 24 | 2010 
15. Shakespeare's Early History Plays: From Chronicle to Stage, Oxford University Press, 2003, p. 197-201.

16. Richard III, éd. Antony Hammond, The Arden Shakespeare, Londres, Methuen, Intro., p. 49-50. Hammond adoptait une solution moyenne, un "panachage» des deux versions auquel les éditeurs se refusent désormais d'une manière générale quelle que soit leur préférence.

17. Dominique Buisset, «Stasimon ", L'Esprit du théâtre, éd. Dominique Goy-Blanquet, Bruxelles, Le Cri/in'hui ${ }^{\circ}$ 64, juillet 2005, p. 28, 31.

18. Eschyle, Les Suppliantes, 603-605: e!nispe d' eh9mi=n1 [...] 㨡 dh\&mou kratou=sa xei \r o3ph7plhqu\&netai.

19. Brian Gibbons, "Is Brevity the Soul of Wit ? Copiousness and Shakespeare ", L'Esprit du théâtre, p. 85-105.

20. Richard Marienstras, « Reprendre Virgile : Shakespeare et la matière de Troie ", L'Esprit du théâtre, p. 130-34.

21. Jacques Darras, Nous ne sommes pas faits pour la mort, Paris, Stock, 2006, p. 33.

22. Conférence à l'invitation de l'Université de Tours, avril 2005.

23. Peter Hall, Making an Exhibition of Myself, Londres, Sinclair-Stevenson, 1993, p. 128.

24. Le Roi Lear, mise en scène d'André Engel pour l'Odéon aux Ateliers Berthier, mars 2006. Richard III au Théâtre des Amandiers, Nanterre, septembre 2005, mise en scène Philippe Calvario.

25. Peter Brook, interview par Marion Thébaud, Le Figaro, 29 sept. 2000.

26. Brook, interview par J.-P. Thibaudat, Libération, 30 nov. 2000.

27. Walter Pater (1839-1894), «Shakespeare's English Kings », in Appreciations, 1889, Londres, Macmillan, 1924, p. 201.

28. Lettre du 31 mars 1861, Correspondance, tome Iv (années 1874-1885, addendum), in CEuvres complètes, Paris, Imprimerie nationale, 1952.

29. François Guizot, Shakespeare et son temps, Didier, Paris, 1852, p. 357-362. Son édition révisée de Letourneur paraît entre 1822 et 1830, celle de François-Victor Hugo entre 1859 et 1866.

30. Euvres complètes de W. Shakespeare, trad. François-Victor Hugo, tome XI, "La Patrie, I ", Introduction (Hauteville-house, 31 décembre 1862), Paris, Pagnerre, 1872, p. 7-50.

31. Dans une lettre adressée à «Monsieur le Rédacteur en chef du Figaro" le 14 avril 1864, publiée sans date. Baudelaire, Euvres complètes, éd. Claude Pichois, Paris, Gallimard, Pléiade, 1961, p. 1170.

32. Alexandre Dumas Père et la Comédie française, éd. Fernande Bassan, Paris, 1972.

33. Dans sa lettre du 13 août 1776 à D'Alembert sur le "préconiseur » de Shakespeare, Letourneur.

34. «General Introduction ", A Textual Companion, p. 15.

35. Le Théâtre et son double, Préface, in Cuvres complètes, vol. IV, Paris, Gallimard, NRF, 1964 (1938), p. 18.

36. «En finir avec les chefs d'œuvre », op. cit., p. 89, 92, 94, 100.

37. Cette « Theatre of Cruelty Season » en 1964 culmine avec le Marat/Sade de Peter Weiss mis en scène par Brook.

38. Hall, op. cit., p. 175-76.

39. Par l'acteur Hugh Quarshie.

40. The Wars of the Roses, Adapted for the Royal Shakespeare Company from William Shakespeare's 'Henry VI, Parts I, II, III and Richard III', BBC, Londres, 1970, Intro., p. xi.

41. As You Like It, III.iii.14-16.

42. "Sonnets et poèmes", Introduction, I, p. 22, "Guernesey, Hauteville house, novembre 1856 ", aux Sonnets. Poëmes. Testament, in Euvres complètes de Shakespeare, vol. XV, Paris, Pagnerre, 1873. La réédition de 1880 ne comporte que la section II de l'Introduction, où F.-V. Hugo commente son réarrangement de la séquence narrative. 
43. "Sonnets et poèmes ", Introduction, II, p. 28.

44. Sir Philip Sidney, Astrophel and Stella, I, 14.

45. L'Anthologie bilingue de la poésie anglaise, établie par Paul Bensimon, Bernard Brugière, François Piquet et Michel Rémy, Bibliothèque de la Pléiade, Paris, Gallimard, 2006, est présentée comme « la formation d'une langue sur la barque de l'histoire et du temps ».

46. Henry VIII, I.i.26-28.

47. William Shakespeare, Livre VI, p. 255.

48. Op. cit., Deuxième partie, Livre I, p. 175.

49. La Cité de Dieu, $\mathrm{xI}, \mathrm{xviii}$, citant Corinthiens, vI. 7-10.

50. Words and Poetry, Londres, Hogarth Press, p. 164-65.

51. Yves Bonnefoy, Théâtre et Poésie. Shakespeare et Yeats, Paris, Mercure de France, 1998, p. 220.

\section{RÉSUMÉS}

Pour Aristote, le poète dramatique écrit en vers, cela va de soi, mais ce n'est pas l'essentiel, il " doit être poète d'histoires plutôt que de mètres ». C'est au système des faits qu'on reconnaît le bon poète, non à la qualité de ses vers. Avantage, donc, semble-t-il à l'action sur les «assaisonnements» du langage. Divers éditeurs récents de Shakespeare notent que la poésie du texte entrave son élan dramatique : le poète oublieux des exigences de la scène s'est laissé aller à des débordements verbaux et verbeux qu'il appartient aux acteurs de retailler, condition nécessaire à la "socialisation de la pièce ». Comment se règlent les comptes entre le poète et l'homme de théâtre?

Drama and poetry today have reached such a degree of antagonism that "Shakespeare poet" sounds like an oxymoron. While old Stratfordians deplore the young actors' inability to speak Shakespeare's verse, “'tis too long for a play" serves directors as an argument against poetic elaborations which slow down the action. In the 'fifties, Peter Brook had turned Artaud's attack on written poetry into a war machine against the established theatre. The OUP editors' declared preference for texts that have "both suffered and benefited from direct contact with the theatre" extended the quarrel from the stage to the publishers and the universities. The break was now complete with the $19^{\text {th }}$ century view of Shakespeare's plays as "exquisite poems", a break confirmed by their exclusion from the recent Pleiade anthology of British poetry.

\section{AUTEUR}

\section{DOMINIQUE GOY-BLANQUET}

Goy-Blanquet est professeur à l'Université de Picardie. Elle est auteur de nombreux ouvrages sur le théâtre historique de Shakespeare, de traductions, d'articles pour Esprit, le Times Literary Supplement et la Quinzaine littéraire. Parmi ses derniers livres on citera Shakespeare's Early History Plays : From Chronicle to Stage (O.U.P., 2003), Shakespeare et l'invention de l'histoire (nouvelle édition, Le Cri, Bruxelles, 2004), Richard II, William Shakespeare (Armand Colin, 2004). 